(2) Open Access Full Text Article

\title{
Antitumor activity of pluripotent cell-engineered vaccines and their potential to treat lung cancer in relation to different levels of irradiation
}

This article was published in the following Dove Press journal:

OncoTargets and Therapy

II March 2016

Number of times this article has been viewed

\author{
Yan-na Zhang \\ Xiao-gang Duan \\ Wen-hui Zhang \\ Ai-ling Wu \\ Huan-Huan Yang \\ Dong-ming $\mathrm{Wu}$ \\ Yu-Quan Wei \\ Xian-cheng Chen
}

State Key Laboratory of Biotherapy/ Collaborative Innovation Center for Biotherapy, West China Hospital, West China Medical School, Sichuan University, Chengdu, Sichuan Province, People's Republic of China
Correspondence: Xian-cheng Chen State Key Laboratory of Biotherapy/ Collaborative Innovation Center for Biotherapy, West China Hospital, West China Medical School, Sichuan University, No 17, South Ren Min Road Third Section, Wu Hou Zone, Chengdu, Sichuan Province 61004I, People's Republic of China Tel +86 I89 80083598

Email chenwcrs@I26.com

\begin{abstract}
Cancer stem cells (CSCs) are critical for tumor initiation/maintenance and recurrence or metastasis, so they may serve as a potential therapeutic target. However, CSC-established multitherapy resistance and immune tolerance render tumors resistant to current tumor-targeted strategies. To address this, renewable multiepitope-integrated spheroids based on placentaderived mesenchymal stem cells (pMSCs) were X-ray-modified, at four different irradiation levels, including 80,160, 240, and $320 \mathrm{~Gy}$, as pluripotent biologics, to inoculate hosts bearing Lewis lung carcinoma (LL2) and compared with X-ray-modified common LL2 cells as control. We show that the vaccines at the 160/240 Gy irradiation levels could rapidly trigger tumor cells into the apoptosis loop and evidently prolong the tumor-bearing host's survival cycle, in contrast to vaccines irradiated at other levels $(P<0.05)$, with tumor-sustaining stromal cell-derived factor-1/CXCR4 pathway being selectively blockaded. Meanwhile, almost no or minimal toxicity was detected in the vaccinated hosts. Importantly, 160/240 Gy-irradiated vaccines could provoke significantly higher killing of CSCs and non-CSCs, which may provide an access to developing a novel biotherapy against lung carcinoma.
\end{abstract}

Keywords: lung carcinoma, placenta-derived mesenchymal stem cells (pMSCs), attenuated vaccine, irradiation level

\section{Introduction}

Many human cancers remain incurable due to the inability of current approaches to target cancer stem cells (CSCs, the renewable cell subsets responsible for tumor initiation, maintenance, and recurrence or metastasis). CSC-established survival pluripotency renders tumors resistant to peripheral lymphocyte reactivity as well as conventional anticancer strategies, including radiation, chemotherapy, and smallmolecule targeted therapies. Lung carcinoma, one of the most widespread malignant tumors in developed countries and the leading cause of cancer death worldwide today, is poised to execute $\sim 1$ billion lives during the 21 st century. ${ }^{1}$ In recent years, aggressive surgical debulking along with multimodal chemotherapy has therapeutically benefited patients with non-small-cell lung cancer (NSCLC), and newly developed biotherapies along with radiotherapy may be helpful supplements. Despite the limited success of current treatment modalities for NSCLC, a survival plateau has been reached; thus, it is urgent to explore other more effective strategies. Nevertheless, CSC-targeted biologics is one of the most promising biostrategies for future oncotherapies, especially for lung carcinoma administration. Anticancer vaccines might provide an avenue for generating specific antitumor immunity with reduced toxicity and prolonged immune memory and reactivity, ${ }^{2}$ compared with traditional therapies 
(chemotherapy, surgery, radiotherapy, etc); thus, they have gradually emerged as a new growing focus of research on combating lung cancer.

Recent studies ${ }^{3,4}$ have shown that placenta-derived mesenchymal stem cells ( $\mathrm{pMSCs}$ ) could modulate immune activity via pluripotent interaction between cells and cytokine networks involving $\mathrm{T}$-cell proliferation and response, which may be more suitable for the biologics used for antitumor immunity, through developing the function of immune reconstruction. Especially, there are very similar biological features and multipotent properties in pMSCs and CSCs. ${ }^{5}$ It has been reported that X-ray amelioration could lead to phosphorylation and membrane translocation of calreticulin and further enhance immunogenicity and cross-reactivity of pMSCs to CSCs; yet, high-dose irradiation might cause too strong phosphatidylserine exposure, which could lead to poor immunogenicity. ${ }^{6}$ Stronger immunogenicity can produce better antitumor reactivity; 7,8 thus, optional irradiation levels for stem cell-engineered biologics might contribute to induction of admirable antitumor effects. In our study, pMSCs were subjected to various levels of irradiation for determining the appropriate irradiation dose so as to develop, in the near future, an ameliorative pluripotent biologic to resist lung cancer progression.

\section{Methods}

\section{Cell isolation, culture, and vaccine preparation}

Fetal deciduous placental tissues were obtained from a healthy mother after informed consent. The pMSCs were isolated and expanded according to a previously reported protocol, ${ }^{9}$ and these were cultured in low-glucose Dulbecco's Modified Eagle's Medium (DMEM; Thermo Fisher Scientific, Waltham, MA, USA) containing 10\% fetal bovine serum (Thermo Fisher Scientific), penicillin (100 U/mL), and streptomycin $(100 \mu \mathrm{g} / \mathrm{mL})$. Purified pMSCs at passages $8-10$ and grown to $85 \%-90 \%$ confluency (Figure S1A and B) were subjected to $80,160,240$, and 320 Gy irradiation using RS-2000 biological irradiator (RadSource), then stored frozen at $-80^{\circ} \mathrm{C}$ for the entire series of experiments. Lewis lung carcinoma (LL2) cell line was obtained from American Type Culture Collection (Manassas, VA, USA) and cultured in DMEM/F12 integrative medium (Thermo Fisher Scientific) for spheroid formation with stem cell-like transition. The spheroid-bearing LL2 cells at logarithmic phase were harvested and irradiated for the experiments (Figure S2A-E).

\section{Animal care}

C57 female mice with average body weight in the range of 16-20 g and aged 5-7 weeks were obtained from the experimental animal center of West China Medical Center of Sichuan University. All mice were housed in air-filtered laminar flow cabinets at a room temperature of $23^{\circ} \mathrm{C} \pm 1^{\circ} \mathrm{C}$ and relative humidity of $45 \% \pm 15 \%$, under a 12 hours $/ 12$ hours ( 7.00 am to $7.00 \mathrm{pm}$ ) light/dark cycle, and fed with AIN-93M rodent diet and autoclaved reverse osmosis-treated water to ensure proper health and living environments before study initiation. All experimental procedures were approved by the Experimental Animal Ethics Committee of Sichuan University. All animalhandling procedures were performed according to the Guide for the Care and Use of Laboratory Animals of the National Institutes of Health and followed the guidelines of the Animal Welfare Act. Experimental mice were randomly assigned to nine study groups (one control and eight experimental groups: LL2 80 Gy, LL2 160 Gy, LL2 240 Gy, LL2 320 Gy; pMSC 80 Gy, pMSC 160 Gy, pMSC 240 Gy, and pMSC 320 Gy) with 20 animals in the control group and ten animals in each experimental group. The control group and experimental groups were subjected to corresponding inoculation and vaccination regimens.

\section{Inoculation regimen and tumor measurement}

In the nine study groups, mice were inoculated with $2 \times 10^{5}$ LL2 cells in the left hind flank at day 0 . On day 7 after tumor inoculation, the eight experimental groups of mice were separately immunized three times at 1 -week intervals with $10^{5}$ irradiated LL2 and pMSC cells in $0.1 \mathrm{~mL}\left(10^{6}\right.$ cells $\left./ \mathrm{mL}\right)$ by subcutaneous injection into the right hind flank, while the control group was immunized three times at the same intervals with physiological saline. After immunization, according to the actual growth of the tumor, A (the largest dimension) and $\mathrm{B}$ (the smallest dimension) of the tumor were measured using a Vernier caliper to judge the inhibition of tumor growth. Tumor volume was determined using the following formula: tumor volume $\left(\mathrm{mm}^{3}\right)=1 / 2 \times A(\mathrm{~mm}) \times$ $\mathrm{B}^{2}\left(\mathrm{~mm}^{2}\right)$, which is represented as volume \pm standard deviation (SD). ${ }^{10}$

\section{Magnetic bead microarray and Western blotting}

Nonnecrotic fresh tumors or tumor-free local inoculum were cut into small pieces, homogenized in liquid nitrogen, melted in moderate-strength radioimmunoprecipitation assay (RIPA) lysis buffer (Beyotime, Nantong, Jiangsu, People's 
Republic of China), blended with protease inhibitor, RIPA added to make up $100 \mu \mathrm{L}$ RIPA per $10 \mathrm{mg}$ tumor tissue, then cracked 30 minutes on ice, centrifuged $(12,000 \mathrm{rpm}$, 20 minutes, $4^{\circ} \mathrm{C}$ ) after fully cracking, supernatant separated and stored at $-80^{\circ} \mathrm{C}$; aliquots of the same were used for detection of cytokine and chemokine expression using the MILLIPLEX ${ }^{\circledR}$ MAP mouse cytokine/chemokine magnetic bead panel kit (Luminex Corporation, Austin, TX, USA) and Luminex 200 microarray analysis system (Luminex Corporation). CXCR4, stromal cell-derived factor (SDF)-1, phosphorylated AKT (P-AKT), and AKT2 levels were determined by Western blotting using $15 \mu \mathrm{g}$ extracted proteins from each sample. Furthermore, $\beta$-actin (diluted 1:1,000) and glyceraldehyde-3-phosphate dehydrogenase (GAPDH; diluted 1:500; EMD Millipore, Billerica, MA, USA) were used as internal controls.

\section{Histomorphometry and immunohistochemistry analysis}

Tumors were fixed using 4\% paraformaldehyde ( $>24$ hours) for paraffin embedding. Semi-serial sections from the paraffinembedded blocks were taken $4 \mu \mathrm{m}$ apart. Then, sections were stained with hematoxylin and eosin (H\&E) to evaluate tissue viability and quality according to previous protocols and assessed by immunohistochemistry as described. ${ }^{11}$ Tissue sections were deparaffinized and rehydrated by passing through graded alcohols $(100 \%, 95 \%, 85 \%$, and $75 \%)$ and distilled water. Tissue sections were boiled in citrate buffer at high temperature in a pressure cooker for antigen retrieval and treated with 3\% hydrogen peroxide to block endogenous peroxidase activity. The slides were incubated with normal goat serum at $37^{\circ} \mathrm{C}$ and then incubated with the primary antibody at $4{ }^{\circ} \mathrm{C}$ overnight, including mouse monoclonal SDF-1 (diluted 1:80; Abcam, Cambridge, MA, USA) and AKT2 (diluted 1:150; Abcam), as well as rabbit anti-mouse (polyclonal) CXCR4 (diluted 1:50; Abcam) and P-AKT (diluted 1:1,200; Abcam). The tissues were inoculated with the biotinylated goat anti-mouse or goat anti-rabbit secondary antibody (ZSGB-BIO Ltd, Beijing, People's Republic of China) and labeled with the avidin-biotin complex. Next, immunoperoxidase staining procedure was performed using the $3,3^{\prime}$-diaminobenzidine coloration and timely termination method. Counterstaining was done with Harris hematoxylin, then dehydration with graded alcohol, and sealing of the slides using neutral balsam. Distinct cytoplasm with brown staining was accepted as a positive result, which was evaluated by the Quantimet 600 image analysis system and Qwin software (Leica Microsystems, Wetzlar, Germany).

\section{In vitro protective response assay for humoral defense alteration by flow cytometry}

The peripheral serum was harvested 1 week after the final booster immunization and pooled for each group. The potential of peripheral serum from different irradiation dose groups to bind to LL2 tumor-initiating spheroid cells was detected using serum diluted 1:50 in $200 \mu \mathrm{L}$ versus $10^{5}$ target cells. The serum-binding index was calculated as a ratio of number of binding-positive cells to total cells, evaluated by dynamic flow cytometry immunofluorescence.

\section{Interleukin-2 assay of peripheral serum by enzyme-linked immunosorbent assay}

Additional peripheral serum was used for interleukin (IL)-2 assay using mouse IL-2 enzyme-linked immunosorbent assay (ELISA) kit (WuHao Trade Co, Ltd, Shanghai, People's Republic of China) according to the manufacturer's instructions. Mean absorbance was calculated from duplicates for each serum dilution that yielded half-maximal binding. The optical density of each well was read within 30 minutes with a microplate reader set to $450 \mathrm{~nm}$ following the assay procedure manual.

\section{Apoptosis analysis}

Apoptosis was observed by Hoechst 33258 staining (Apoptosis-Hoechst staining kit; WuHao Trade Co, Ltd Biotechnology). Briefly, paraformaldehyde-fixed sections were deparaffinized, rehydrated, and treated with $2 \times$ standard saline citrate (SSC) at $80^{\circ} \mathrm{C}$ for 20 minutes, double distilled water $\left(\mathrm{ddH}_{2} \mathrm{O}\right)$ at room temperature for 10 minutes, and $0.1 \mathrm{M}$ Tris/ $0.05 \mathrm{M}$ ethylenediaminetetraacetic acid (EDTA) at $42^{\circ} \mathrm{C}$ for 2 minutes. Sections were incubated with Hoechst 33258 in a dark humid chamber for 20 minutes at room temperature, rinsed twice in phosphate-buffered saline, and mounted with SlowFade ${ }^{\circledR}$ Light Antifade Reagent (Molecular Probes, Eugene, OR, USA) prior to viewing via excitation $348 \mathrm{~nm} /$ emission $480 \mathrm{~nm}$ wavelengths.

\section{Statistical analyses}

Data are expressed as arithmetic mean \pm SD. Mean values were compared using Student's $t$-test. Differences in time to progression and survival were analyzed using the KaplanMeier method, and groups were compared using the log-rank test with one-way analysis of variance by SPSS 16.0 software. $P<0.05$ was considered to be statistically significant at a $95 \%$ confidence interval. 
A

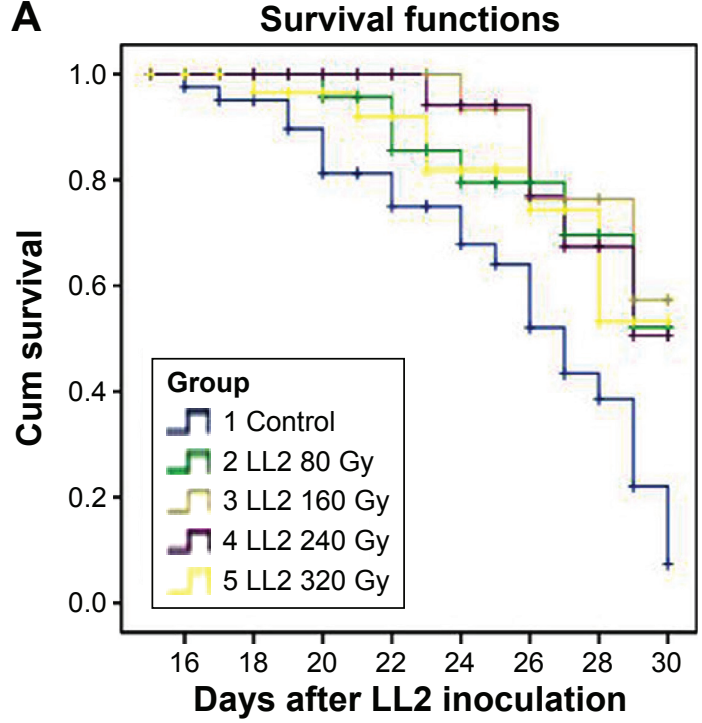

C

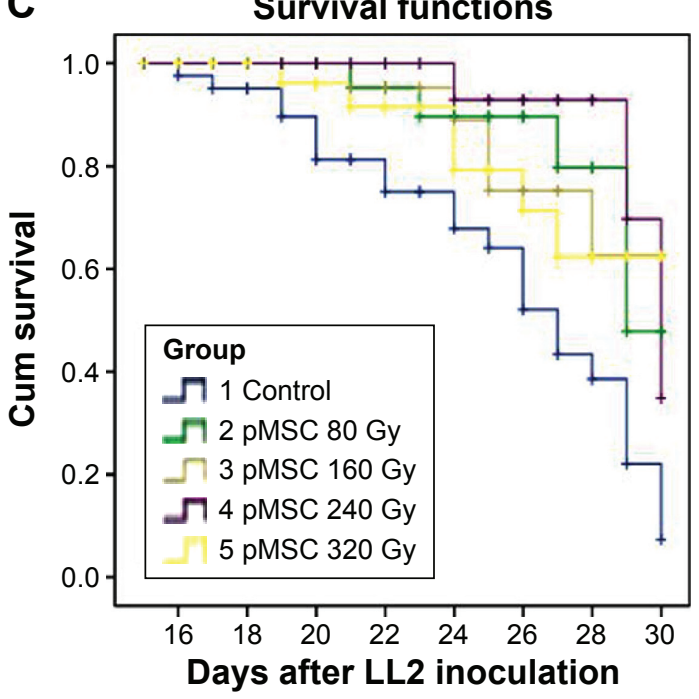

B

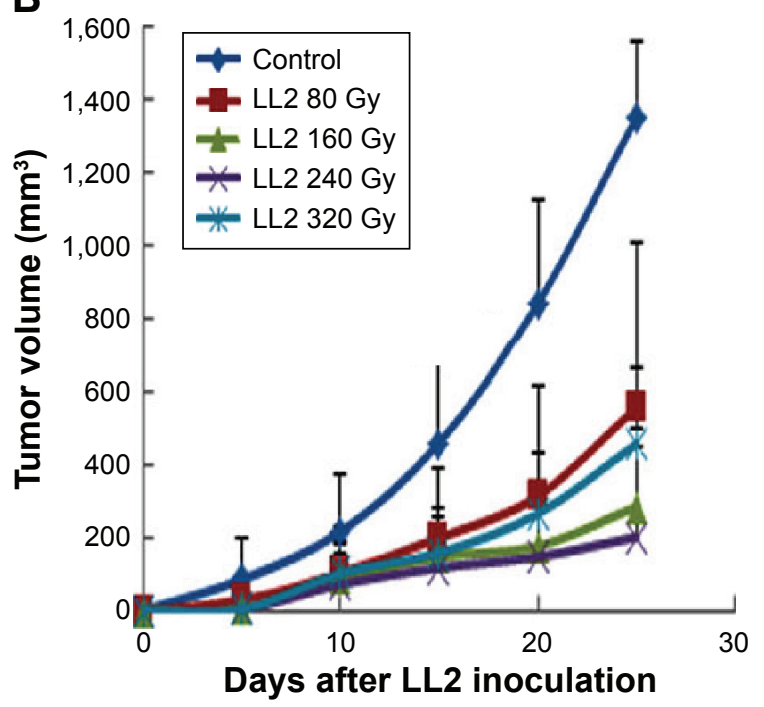

D

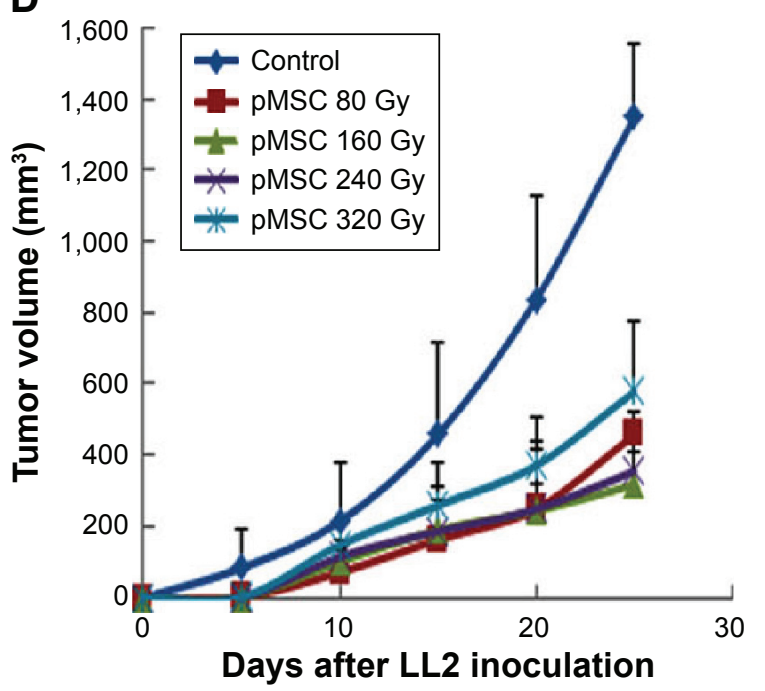

Figure I Effects of various dose-irradiated pluripotent vaccines on tumor progression and host survival.

Notes: As the cumulative (Cum) host survival (A and C) and tumor development (B and D) curves show, 160 and 240 Gy pluripotent vaccines, particularly I60 Gy, could significantly impede LL2 in vivo progression in the tumor-bearing mice, leading to preferable elongation of host survival versus the control and other groups (Kaplan-Meier analysis, $P<0.05$ ). LL2 tumor developmental and progressive dynamics are extensively reversed in the final stage by $160 / 240 \mathrm{~Gy}$ vaccines, but not by other vaccines or control inoculation.

Abbreviations: Cum, cumulative; LL2, Lewis lung carcinoma; pMSC, placenta-derived mesenchymal stem cell.

\section{Results}

Pluripotent cell-engineered vaccines suppressed lung cancer growth and prolonged host survival cycle

As shown in Figure 1A and C, 160 and 240 Gy vaccines could lead to preferable survival of mice during the observation period, whereas the others yielded low survival levels (Kaplan-Meier analysis; $P<0.05$ ). Yet, there was no statistical significance between 160 and 240 Gy groups $(P>0.05)$. As the tumor development (Figure 1B and D) curves show, 160 and 240 Gy-irradiated vaccines could significantly attenuate the growth of tumor as compared to that seen in other groups during the observation period $(P<0.05)$. Tumor size in 160 and 240 Gy groups was much smaller than in the other groups. LL2 tumor developmental and progressive dynamics was extensively reversed in the final stage by $160 / 240$ Gy vaccines, not by other vaccines or after control vaccination.

Sequential histomorphometry assay with H\&E/Hoechst double stain showed that $160 / 240$ Gy-irradiated vaccines could selectively drive in vivo resistant LL2 subsets into apoptosis. Thus, the anticancer resistance of the in vivo LL2 pool was actually reversed in the 160/240 Gy group, 


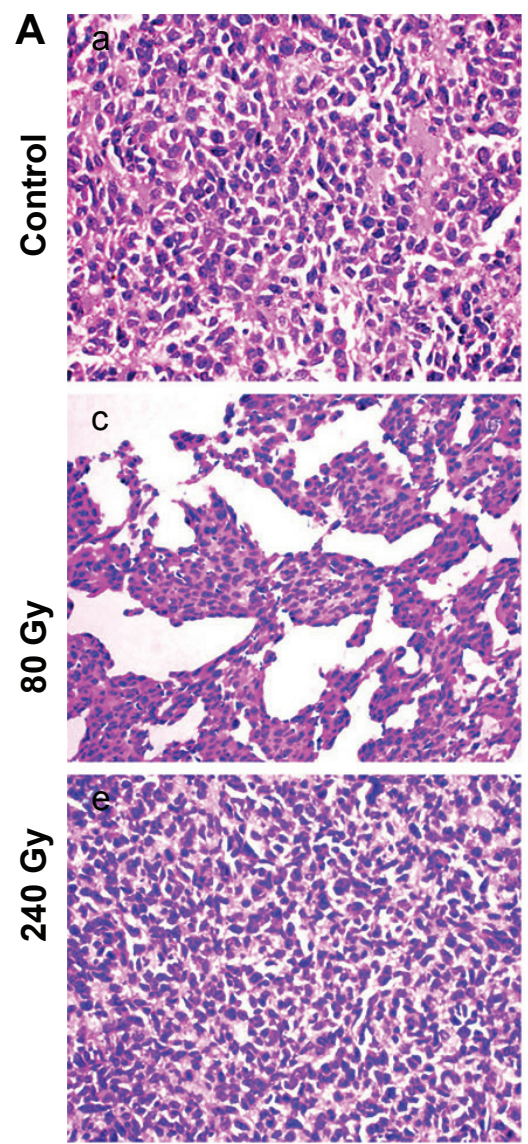

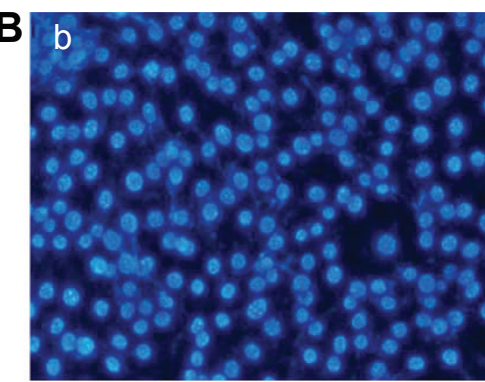
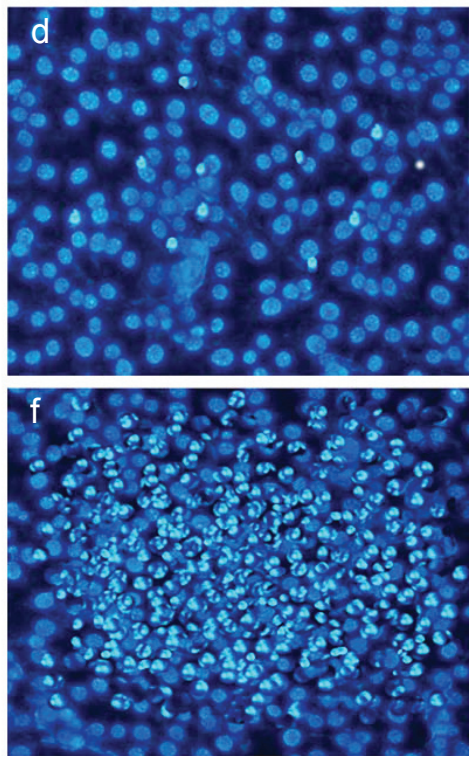
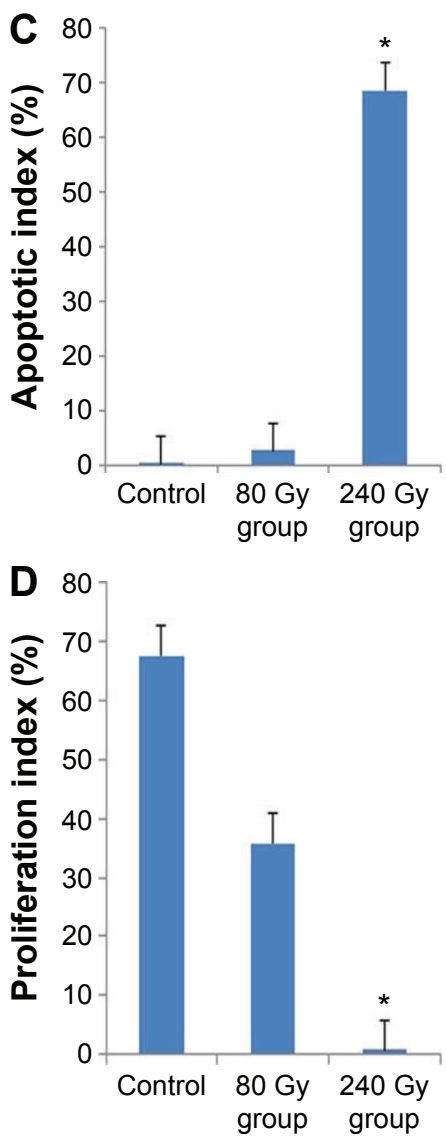

Figure 2 Pluripotent vaccines could drive in vivo LL2 cells into apoptosis in the tumor-bearing hosts.

Notes: Sequential histomorphometry assay with H\&E/Hoechst double stain showed that 160/240 Gy-irradiated vaccines could selectively drive resistant LL2 subsets into apoptosis in vivo. Thus, (A and B) the anticancer resistance of in vivo LL2 pool was actually reversed in the 160/240 Gy groups, but not in the control and 80 Gy groups. (C and D) The final apoptosis and proliferation index analysis showed no appreciable differences between the control group and the $80 \mathrm{~Gy}$ group; only the I60/240 Gy vaccines resulted in significant increment of apoptotic index and decrease of proliferation index of in vivo LL2 cells. Experiment was repeated in the pMSC vaccine groups with similar results as in the LL2 vaccine groups. Values are expressed as average apoptotic index $\pm S D(* P<0.05$ versus other groups, respectively). Original magnifications: $\times 200$ (A and $\mathbf{B})$.

Abbreviations: H\&E, hematoxylin and eosin; LL2, Lewis lung carcinoma; pMSC, placenta-derived mesenchymal stem cell; SD, standard deviation.

but not in the control and 80 Gy groups (Figure 2A and B). The final apoptosis and proliferation index analysis showed no appreciable differences between the control group and the 80 Gy group; only the $160 / 240$ Gy vaccines resulted in significant increment of the apoptotic index and decrease in proliferation index of in vivo LL2 cells (Figure 2C and D). The experiment was repeated in the pMSC vaccine groups with similar results as in the LL2 vaccine groups. Values are expressed as average apoptosis index $\pm \mathrm{SD}\left({ }^{*} P<0.05\right.$ versus other groups, respectively), with $\times 200$ magnification of tissue sections.

Histomorphometry and immunohistochemistry analysis showed that SDF-1/CXCR4 and AKT2/p-AKT signal molecules, although retaining active expression in control and 80 Gy groups, were depleted or lost in 160/240 Gy-irradiated groups (Figure 3A), leading to loss of LL2 renewal signaling. Meanwhile, Western blotting
(Figure 3B) of tumor tissue extracts showed that tumorgoverned SDF-1/CXCR4-AKT2/p-AKT signal loop, though showing active expression in control and 80 Gy groups, declined evidently or was lost in the 160/240 Gyirradiated groups under reprogramming cellular and molecular microenvironment. These alterations in expression verify that the mitogen-activated protein kinase MAPK-PI3K (phosphoinositide 3-kinase)/protein kinase $\mathrm{B}$ (AKT)-NF- $\mathrm{BB}$ (nuclear factor-kappa B) pathway plays a crucial role in LL2 tumor progression or retrogression.

\section{Pluripotent vaccines renovate humoral defense microenvironment with peripheral serum binding to LL2 cells}

To test whether alterations in the irradiation dose cause humoral defense variance after immunization, circulating peripheral serum from the control and experimental groups 

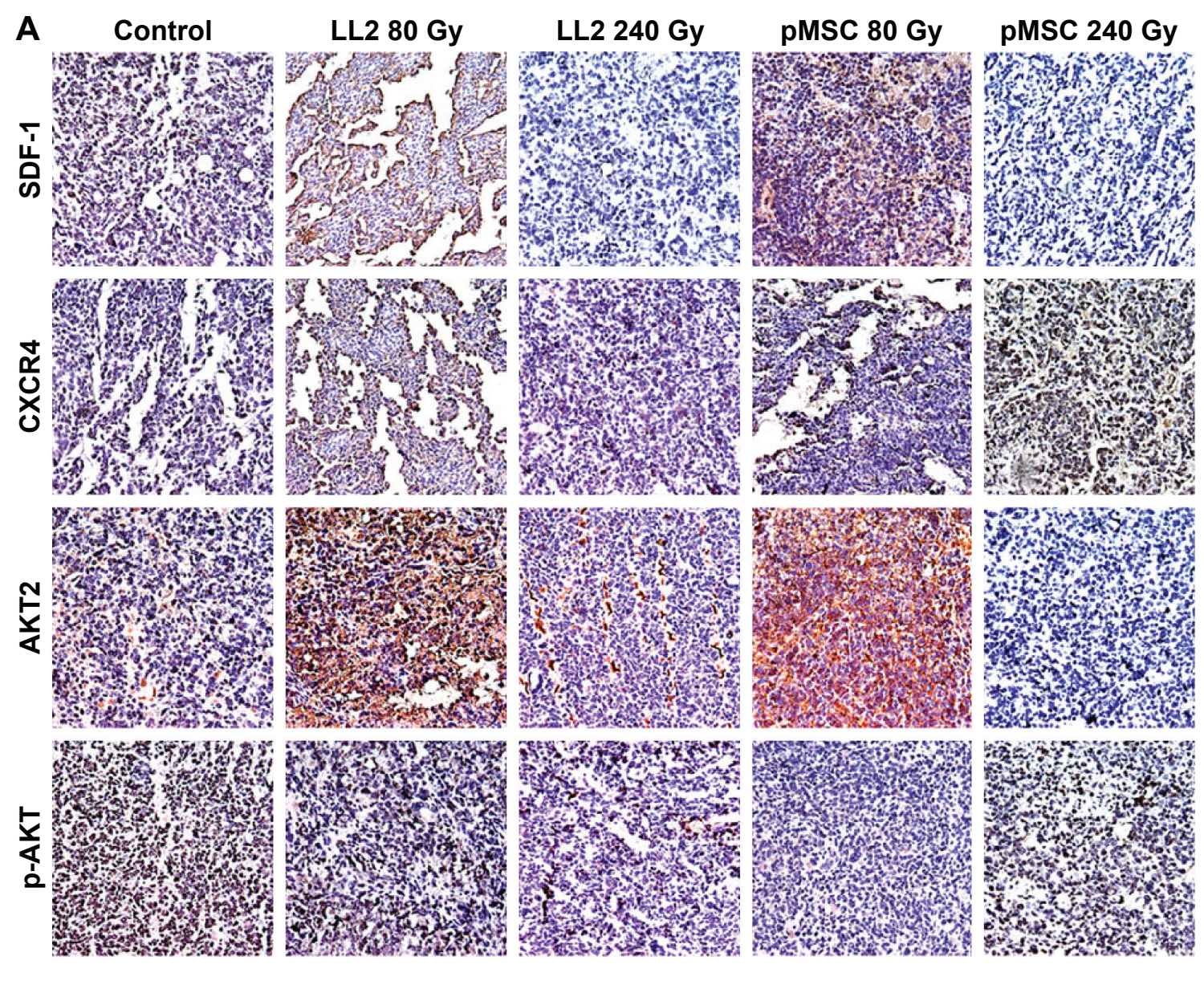

B

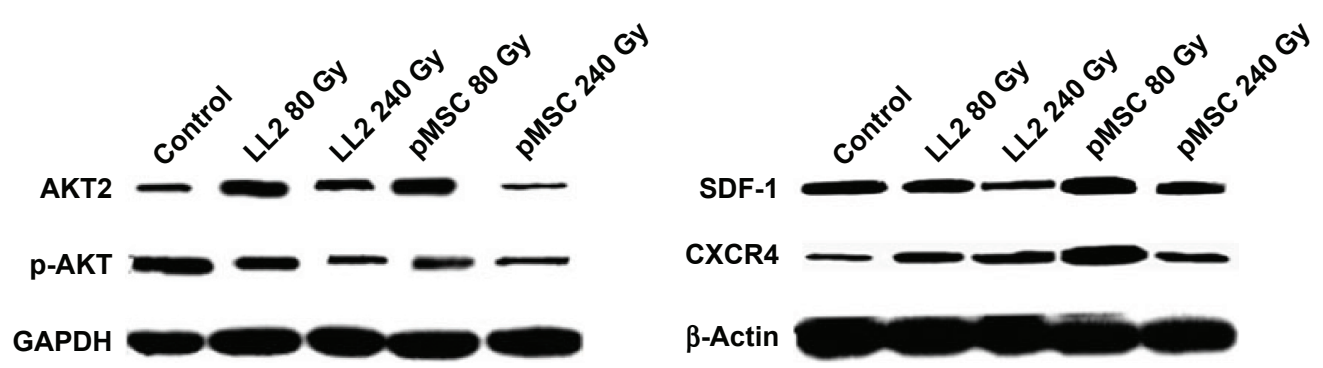

Figure 3 Tumor-sustaining SDF-I/CXCR4-AKT2/P-AKT loop could be depleted by pluripotent vaccines with consequent tumor retrogression.

Notes: (A) Histomorphometry and immunohistochemistry analysis have shown that SDF-1/CXCR4 and AKT2/p-AKT signal molecules, though showing active expression in control and 80 Gy groups, have been depleted or lost in the 160/240 Gy-irradiated groups (magnification: $\times 200$ ), leading to loss of LL2 renewal signaling. (B) Meanwhile, Western blotting for the tumor tissue extracts shows that the tumor-governed SDF-I/CXCR4-AKT2/p-AKT signal loop, though showing active expression in control and $80 \mathrm{~Gy}$ groups, has declined evidently or is lost in the 160/240 Gy-irradiated groups under reprogramming cellular and molecular microenvironment. These alterations in molecular expression show that the MAPK-PI3K/AKT-NF-KB pathway plays a crucial role in LL2 tumor progression or retrogression.

Abbreviations: SDF, stromal cell-derived factor; AKT, protein kinase B; LL2, Lewis lung carcinoma; MAPK, mitogen-activated protein kinase; NF- $K B$, nuclear factor-kappa B; PI3K, phosphoinositide 3-kinase; pMSC, placenta-derived mesenchymal stem cell; GAPDH, glyceraldehyde-3-phosphate dehydrogenase; CXCR, chemokine receptor.

was collected on the seventh day after the third immunization, and these samples were tested by flow cytometry for the potential of binding to LL2-initiating cells/CSCs. Flow cytometry analysis showed that the potential of binding to LL2 cells had obviously risen in the experimental groups (Figure 4; $P<0.05$ ) compared to the control. Meanwhile, the results for the binding experiment were different among various irradiation levels, with the most obvious increment observed in the $160 / 240$ Gy-irradiated groups. Thus, the humoral defense microenvironment was renovated by pluripotent vaccines with peripheral serum targeting LL2initiating cells.

\section{Irradiation dose alteration could lead to T-cell growth factor IL-2 variance}

ELISA for peripheral serum showed that the cytokine IL-2 was significantly enhanced by 160/240 Gy-irradiated vaccines, with a concentration as high as 4.582 (pMSC 


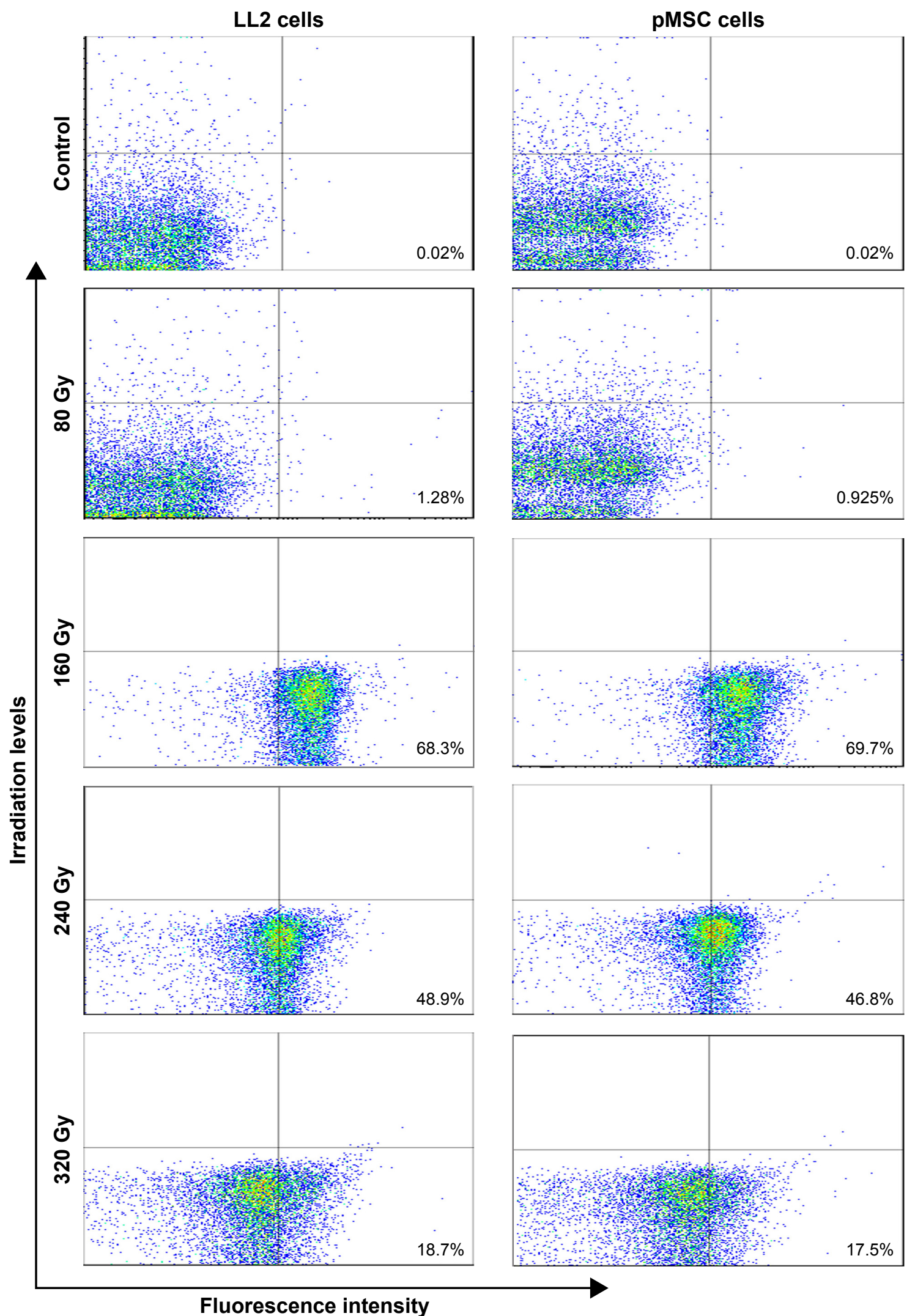

Figure 4 Humoral defense microenvironment renovated by pluripotent vaccines with serum binding to LL2 initiating cells.

Notes: The potential of peripheral serum binding to LL2-initiating cells/CSCs was detected by flow cytometry. Percentage of positive cells for each histogram is shown respectively. The result showed that pluripotent vaccines with varying irradiation levels may cause evident amelioration of humoral defense microenvironment targeting LL2-initiating cells, especially in the 160/240 Gy-immunized groups.

Abbreviations: CSC, cancer stem cell; LL2, Lewis lung carcinoma; pMSC, placenta-derived mesenchymal stem cell. 


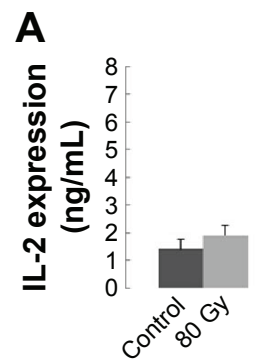

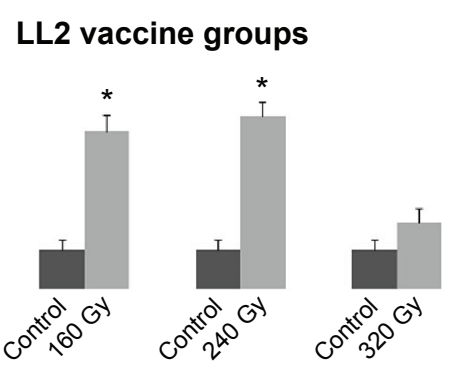

Irradiated dose

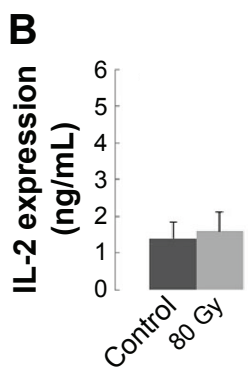

Figure 5 Pluripotent vaccines could enhance T-cell growth factor IL-2 of peripheral serum.

Notes: LL2 vaccines (A), PMSC vaccines (B). ELISA for peripheral serum showed that cytokine interleukin-2 (IL-2) is significantly enhanced in the final stage of the immune response process by integrative microenvironments amelioration using $160 / 240$ Gy vaccines, but not by control inoculation (*P<0.0I versus control). Reactivity in the 160/240 Gy-immunized vaccine groups was stronger than that in the other experimental groups. Values expressed as mean \pm SD.

Abbreviations: ELISA, enzyme-linked immunosorbent assay; IL, interleukin; LL2, Lewis lung carcinoma; pMSC, placenta-derived mesenchymal stem cell.

vaccine groups) and $6.235 \mathrm{ng} / \mathrm{mL}$ (LL2 vaccine groups) after the third immunization (Figure $5 ; P<0.01$ versus control). The reactivity in the 160/240 Gy-immunized vaccine groups was stronger than in the other experimental groups. Nonetheless, there was no statistical significance between 160 and 240 Gy groups $(P>0.05)$. The study suggested that IL-2 is significantly enhanced by integrative microenvironment amelioration using 160/240 Gy-irradiated vaccines, but not by control inoculation, in the final stage of the immune response process.

\section{Expression of immunoregulatory factors in pluripotent cell vaccine groups}

The expression of various immunoregulatory cytokine/ chemokine factors in the experimental groups and the controls were analyzed by magnetic bead microarray using Luminex 200 microarray analysis system (Luminex Corporation). The 160 Gy-LL2/240 Gy-pMSC groups demonstrated enhanced elaboration of key immunoregulatory factors of the interferon (IFN)- $\gamma /$ T-reactivating loop, including IP-10, IL-4, IL-7, IL-12, IL-17, IFN- $\gamma$, monokine induced by interferon- $\gamma$ (MIG), Leukemia inhibitory factor (LIF), regulated upon activation in normal $\mathrm{T}$ cell expressed and secreted (RANTES) $(P<0.05)$. Concomitant cross-downregulation of immunosuppressive factors, including vascular endothelial growth factor (VEGF) and MCP-1 (Figure 6A and B), was also detected respectively ( $P<0.05$ versus control). These results support the deduction that 160/240 Gy-irradiated vaccines trigger integral immune system readily and indicate the integrative resuscitation of the molecular defense microenvironment involving the antitumoral reactivity, so that the survival cycle of the tumor-bearing hosts could be effectively prolonged.

\section{Discussion}

Up to now, diverse strategies have been tried to eliminate antitumor resistance, but most human cancers remain incurable mainly due to the lack of suitable pluripotent therapeutic options to address the constantly evolving CSCs. Especially, lung carcinoma presents a continuing clinical challenge as the therapeutic effects of the modalities available are still limited. Therefore, it is sensible to exploit an innovative and safe pluripotent strategy aimed at the development of lung carcinoma.

A previous study ${ }^{12}$ has demonstrated that cytokine/ radiation-engineered MSCs could be tactfully induced by progressive tumor microenvironment to differentiate selectively into stromal cells in tumor sites, but not in normal tissues, and finally be incorporated into the tumor architecture. The engineered MSCs were better capable of migrating to the tumor sites to contribute to long-term selective expression or accumulation within tumor microenvironments by the MSCs homing into tumor sites. ${ }^{12-15}$ It is speculated that a pluripotent antitumor approach with more selective toxicity to tumor cells would be better able to contribute to the maximization of the beneficial therapeutic efficacy on the tumor while minimizing the side effects on the hosts. In our study, 160/240 Gyengineered pluripotent vaccines could significantly impede in vivo LL2 lung cancer growth and progression, with a preferable elongation of survival of the tumor-bearing host. Magnetic bead microarray proved that pluripotent vaccines could evoke the integrative resuscitation of the molecular defense microenvironment, leading to integral cellular/ humoral immune renovation against in vivo LL2 tumor development. The ameliorative microenvironment at the molecular level includes the enhanced elaboration of IP-10, IL-4, IL-7, IL-12, IL-17, IFN- $\gamma$, MIG, LIF, and RANTES, as well as the 

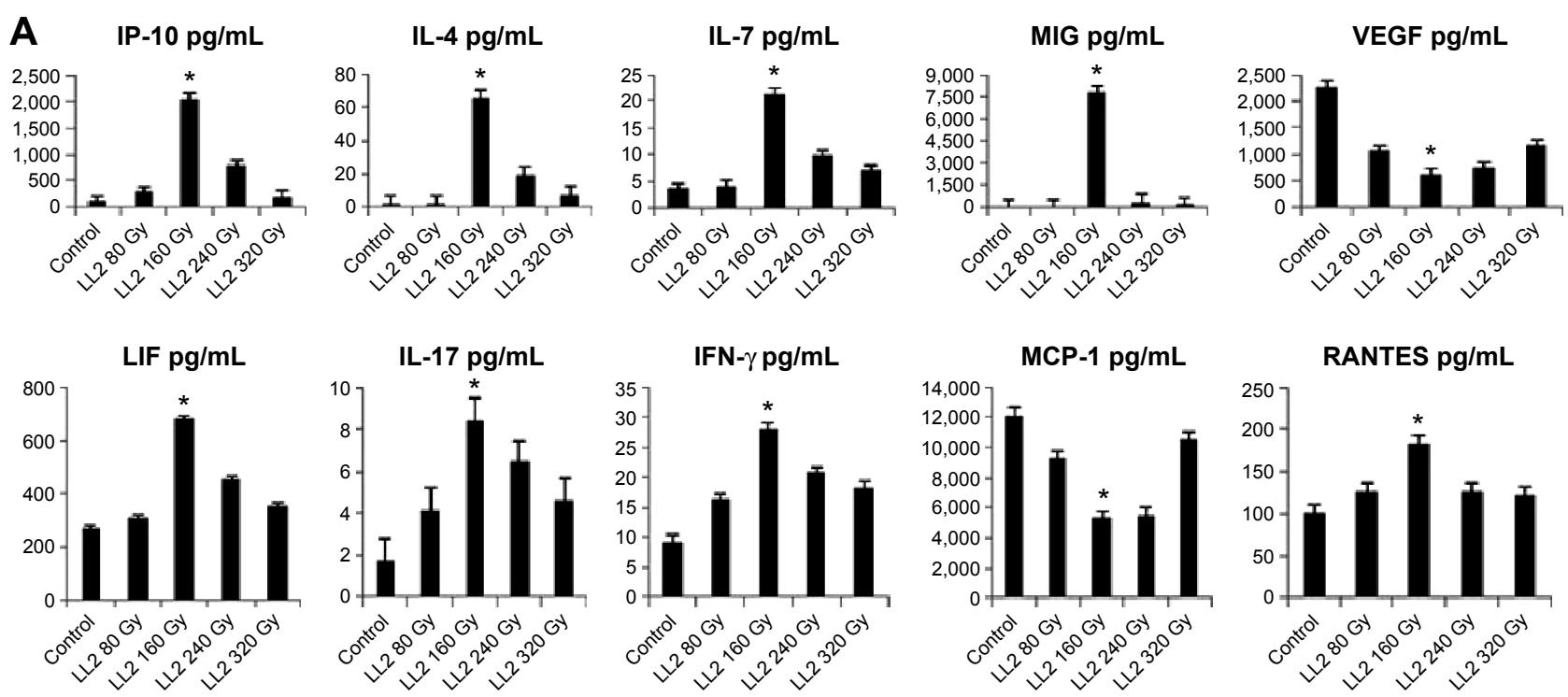

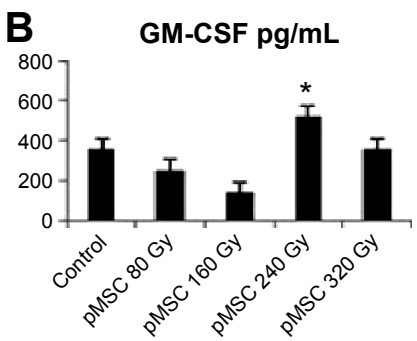

IFN- $\gamma \mathrm{pg} / \mathrm{mL}$

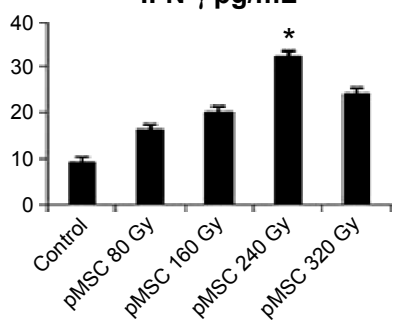

MIG pg/mL

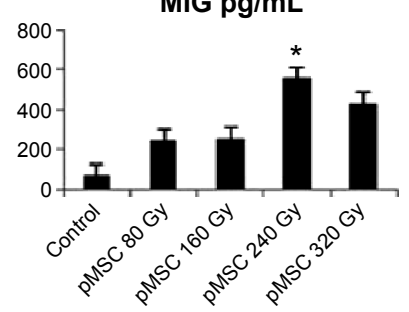

IL-12 (p40) pg/mL

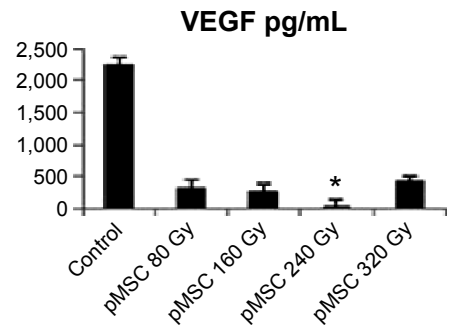

IL-17 pg/mL

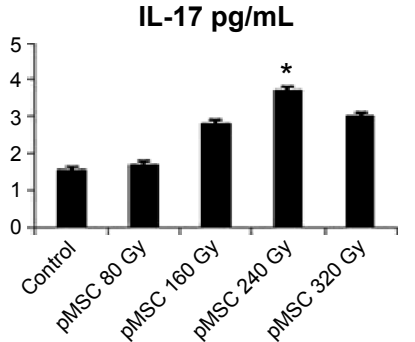

IL-4 pg/mL

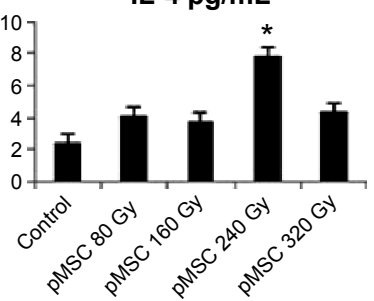

MCP-1 pg/mL

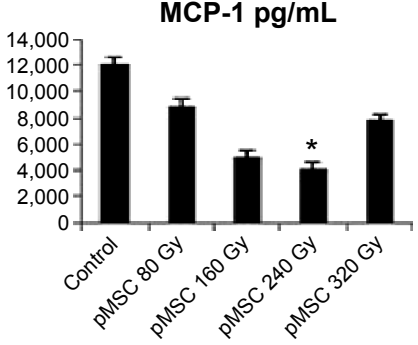

Figure 6 Pluripotent vaccines trigger the integrative resuscitation of molecular defense microenvironment.

Notes: 160Gy-LL2 (LL2 vaccines [A])/240Gy-pMSC (pMSC vaccines [B]) groups particularly demonstrated collaborative elaboration of key immunoregulatory factors of IFN- $/$ /T reactivating loop including IP-I0, IL-4, IL-7, IL-12, IL-17, IFN $\gamma$, MIG LIF, RANTES. Concomitant cross-downregulation of immunosuppressive factors covering VEGF, MCP-I was also detected respectively $(* P<0.05$ versus control).

Abbreviations: IFN, interferon; IL, interleukin-2; IP, IFN- - -inducible protein; LIF, Leukemia inhibitory factor; LL2, Lewis lung carcinoma; MIG, monokine induced by interferon- $\gamma$; MCP, Monocyte chemotactic protein; RANTES, regulated upon activation in normal T cell expressed and secreted; VEGF: vascular endothelial growth factor; GM-CSF, granulocyte-macrophage colony-stimulating factor; SD, standard deviation; PMSC, placenta-derived mesenchymal stem cell.

concomitant cross-downregulation of immunosuppressive factors, including VEGF and MCP-1.

The pluripotent vaccine-evoked integral cellular/ molecular microenvironment renovation could further deplete the tumor-governed SDF-1/CXCR4-AKT2/p-AKT signal loops. Previous studies have identified that $\mathrm{G}$ proteincoupled receptor CXCR4 and its chemokine SDF- $1 \alpha$ play a crucial role in directing migration of tumor cells to neighboring tissues, as well as in metastasis to distant sites via angiogenesis, ${ }^{13,14,16-19}$ whereas CXCL12 (SDF-1) guides the migration of hematopoietic stem cells from fetal liver to bone marrow during embryonic development ${ }^{20,21}$ by binding only to the cognate receptor CXCR4, a specific $\mathrm{G}$ protein-coupled receptor. ${ }^{22,23}$ The intracellular interaction between SDF-1 and CXCR4 could increase NF- $\kappa \mathrm{B}$ activity $^{24-26}$ and evoke the MAPKs. ${ }^{27,28}$ CXCL12/SDF-1, the ligand for CXCR4, could express in the lung and at other sites of metastases. ${ }^{24,29,30} \mathrm{CXCR} 4 / \mathrm{SDF}-1$ could indirectly 
interact with tumor metastasis by enhancing tumor-associated angiogenesis and mediating the proliferation and migration of renewable tumor cells via AKT activation as described earlier. ${ }^{31,32}$ Thus, the pluripotent vaccine-dominated tumoricidal reactivity to refractory lung cancer would be substantially enhanced by the combined renewal and elaboration of the depleted SDF-1/CXCR4-AKT2/p-AKT signal loops and the ameliorated IFN- $\gamma /$ IP-10/MIG/RANTES/IL-2/T-subsets molecule/cellular integrative microenvironments.

In summary, developing an effective immunotherapy for lung cancer patients is a daunting but hugely attractive challenge. CSC-targeted vaccines are the most promising therapeutic approach for lung cancer, which is worth exploring in depth in future studies.

\section{Conclusion}

In summary, pluripotent cell-engineered vaccines modified at the 160/240 Gy irradiation level could significantly reduce tumor burden and achieve improvement of survival in lung carcinoma models. Considering the strong antitumor effect and minimal toxicity, the results of our study may be of importance for exploiting the pluripotent antitumor approach in the treatment of lung carcinoma and other refractory tumors.

\section{Acknowledgments}

This study was supported by the National Natural Science Foundation of China (30973507), the National 973 Basic Research Program of China (2010CB529900), and High-tech Research and Development Program(2014ZX09101041-002). We thank the professors and technologists of National Key Laboratory of Biotherapy, Sichuan University, for their expert technical assistance.

\section{Disclosure}

The authors report no conflicts of interest in this work.

\section{References}

1. Quoix E, Ramlau R, Westeel V, et al. Therapeutic vaccination with TG4010 and first-line chemotherapy in advanced non-small-cell lung cancer: a controlled phase 2B trial. Lancet Oncol. 2011;12(12): 1125-1133.

2. Schuster M, Nechansky A, Kircheis R. Cancer immunotherapy. Biotechnol J. 2006;1(2):138-147.

3. Gu YZ, Xue Q, Chen YJ, et al. Different roles of PD-L1 and FasL in immunomodulation mediated by human placenta-derived mesenchymal stem cells. Hum Immunol. 2013;74:267-276.

4. Liu Q, Zheng H, Chen X, et al. Human mesenchymal stromal cells enhance the immunomodulatory function of $\mathrm{CD} 8 \mathrm{CD} 28$ regulatory $\mathrm{T}$ cells. Cell Mol Immunol. 2014;6:708-718.

5. Huang L, Ma W, Ma Y, Feng D, Chen H, Cai B. Exosomes in mesenchymal stem cells, a new therapeutic strategy for cardiovascular diseases. Int J Biol Sci. 2015;11(2):238.
6. Obeid M, Panaretakis T, Joza N, et al. Calreticulin exposure is required for the immunogenicity of gamma-irradiation and UVC light-induced apoptosis. Cell Death Differ. 2007;14(10):1848-1850.

7. Neeson P, Paterson Y. Effects of the tumor microenvironment on the efficacy of tumor immunotherapy. Immunol Invest. 2006;35(3-4): 359-394.

8. Cassaday RD, Sondel PM, King DM, et al. A phase I study of immunization using particle-mediated epidermal delivery of genes for gp 100 and GM-CSF into uninvolved skin of melanoma patients. Clin Cancer Res. 2007;13(2):540-549.

9. Zheng L, Zhang D, Chen X, Yang L, Wei Y, Zhao X. Antitumor activities of human placenta-derived mesenchymal stem cells expressing endostatin on ovarian cancer. PLoS One. 2012;7:e39332.

10. Lage A, Crombet T, González G. Targeting epidermal growth factor receptor signaling: early results and future trends in oncology. Ann Med. 2002;35(5):327-336.

11. Gorellk E, Segal S, Feldman M. Control of lung metastasis progression in mice: role of growth kinetics of 3LL Lewis lung carcinoma and host immune reactivity. J Natl Cancer Inst. 1980;65(6):1257-1264.

12. Andreeff M, Studeny M, Dembinski J, et al. Mesenchymal stem cells as delivery systems for cancer and leukemia gene therapy. J Clin Oncol. 2004;22:3194-3198.

13. Ceradini DJ, Gurtner GC. Homing to hypoxia: HIF-1 as a mediator of progenitor cell recruitment to injured tissue. Trends Cardiovasc Med. 2005;15(2):57-63.

14. Nakamizo A, Marini F, Amano T, et al. Human bone marrow-derived mesenchymal stem cells in the treatment of gliomas. Cancer Res. 2005; 65(8):3307-3318

15. Ren S-X, Ren Z-J, Zhao M-Y, Wang XB, Zuo SG, Yu F. Antitumor activity of endogenous mFlt4 displayed on a T4 phage nanoparticle surface. Acta Pharmacol Sin. 2009;30(5):637-645.

16. Koshiba T, Hosotani R, Miyamoto Y, et al. Expression of stromal cellderived factor 1 and CXCR4 ligand receptor system in pancreatic cancer: a possible role for tumor progression. Clin Cancer Res. 2000;6(9): 3530-3535.

17. Kryczek I, Lange A, Mottram P, et al. CXCL12 and vascular endothelial growth factor synergistically induce neoangiogenesis in human ovarian cancers. Cancer Res. 2005;65(2):465-472.

18. Hamdy S, Haddadi A, Hung RW, Lavasanifar A. Targeting dendritic cells with nano-particulate PLGA cancer vaccine formulations. Adv Drug Deliv Rev. 2011;63(10):943-955.

19. Vlahakis SR, Villasis-Keever A, Gomez T, Vanegas M, Vlahakis N, Paya CV. G protein-coupled chemokine receptors induce both survival and apoptotic signaling pathways. $J$ Immunol. 2002;169(10): $5546-5554$.

20. Gonzalez G, Montero E, Leon K, Cohen IR, Lage A. Autoimmunization to epidermal growth factor, a component of the immunological homunculus. Autoimmun Rev. 2002;1(1):89-95.

21. Lage A, Perez R, Fernandez L. Therapeutic cancer vaccines: at midway between immunology and pharmacology. Curr Cancer Drug Targets. 2005;5(8):611-627.

22. Crombet Ramos T, Vinageras EN, Catalá Ferrer M, et al. Treatment of NSCLC patients with an EGF-based cancer vaccine: report of a phase I trial. Cancer Biol Ther. 2006;5(2):145-149.

23. Gonzalez G, Lage A. Cancer Vaccines for Hormone Immune-Deprivation: The EGF Vaccine Approach. Leading Topics in Cancer Research. (Chap. 11). New York, NY: Nova Publishers; 2006:293-310.

24. González G, Lage A. Cancer vaccines for hormone/growth factor immune deprivation: a feasible approach for cancer treatment. Curr Cancer Drug Targets. 2007;7(3):229-241.

25. Rodriguez G, Albisa A, Viña L, Cuevas A, Garcia B, García AT. Manufacturing process development for an epidermal growth factorbased cancer vaccine. Biopharm Int. 2008;10:36-42.

26. González G, Crombet T, Neninger E, Viada C, Lage A. Therapeutic vaccination with epidermal growth factor (EGF) in advanced lung cancer: analysis of pooled data from three clinical trials. Hum Vaccin. 2007;3(1):8-13. 
27. García B, Neninger E, de la Torre A, et al. Effective inhibition of the epidermal growth factor/epidermal growth factor receptor binding by anti-epidermal growth factor antibodies is related to better survival in advanced non-small-cell lung cancer patients treated with the epidermal growth factor cancer vaccine. Clin Cancer Res. 2008;14(3): 840-846.

28. Vinageras EN, de la Torre A, Rodríguez MO, et al. Phase II randomized controlled trial of an epidermal growth factor vaccine in advanced non-small-cell lung cancer. J Clin Oncol. 2008;26(9):1452-1458.

29. Neninger E, Verdecia BG, Crombet T, et al. Combining an EGF-based cancer vaccine with chemotherapy in advanced nonsmall cell lung cancer. J Immunother. 2009;32(1):92-99.
30. Degl'Innocenti E, Grioni M, Capuano G, et al. Peripheral T-cell tolerance associated with prostate cancer is independent from CD4+CD25+ regulatory T cells. Cancer Res. 2008;68(1):292-300.

31. Marigo I, Dolcetti L, Serafini P, Zanovello P, Bronte V. Tumor-induced tolerance and immune suppression by myeloid derived suppressor cells. Immunol Rev. 2008;222(1):162-179.

32. He J, Luster TA, Thorpe PE. Radiation-enhanced vascular targeting of human lung cancers in mice with a monoclonal antibody that binds anionic phospholipids. Clin Cancer Res. 2007;13(17):5211-5218. 


\section{Supplementary materials}

A

式

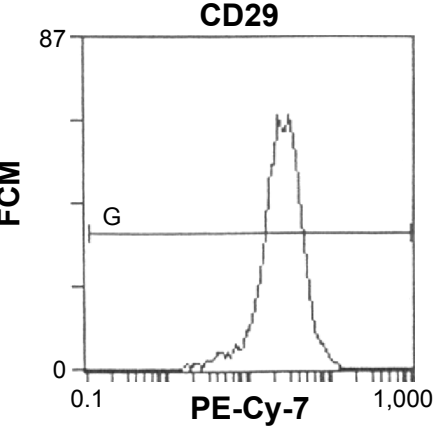

B

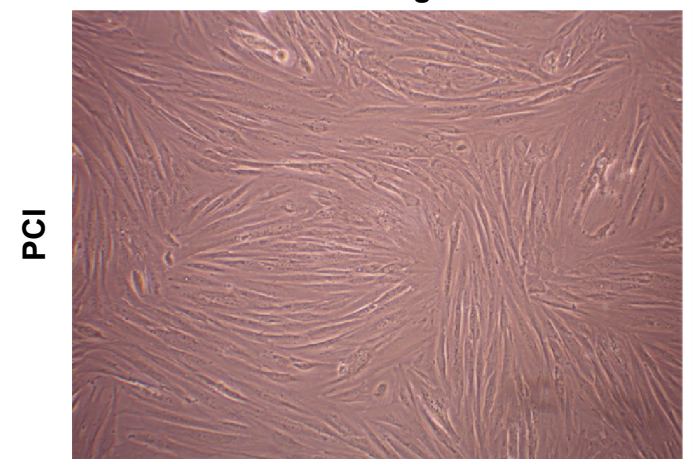

CD44

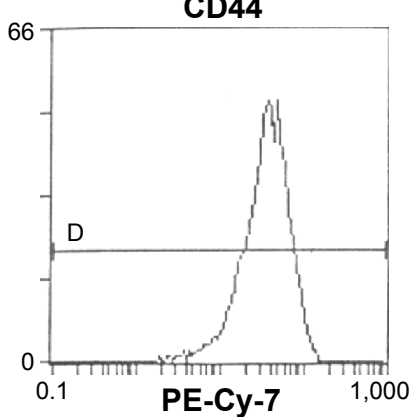

000

Doubling 10
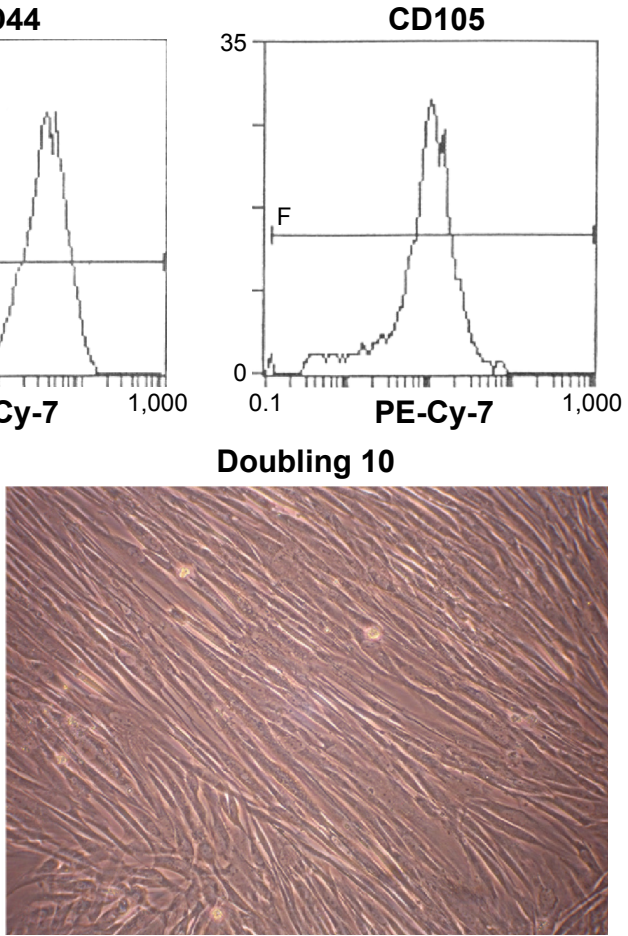

Figure SI Double analyses of FCM and PCI for PMSC phenotype features.

Notes: (A) The phenotype characteristics of purified pMSCs were verified by FCM, which shows that these expanded cell populations are positive for CD29 (>95\%), CD44 (>95\%), and CDI05 (>92\%) as surface markers. (B) The PCI of purified pMSCs at doubling eight and ten exhibited that these plate-adhering cells reached $\sim 85 \%-90 \%$ of confluence and possessed strong growth activity before irradiation for the entire series of experiments. $\times 200$ magnification.

Abbreviations: CD, cell differentiation antigen; FCM, flow cytometry; PCI, phase contrast imaging; PE-Cy-7, phycoerythrin-cyanin-7; pMSC, placenta-derived mesenchymal stem cell.

Pre irradiation

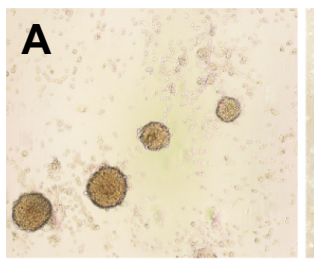

Postirradiation $80 \mathrm{~Gy}$

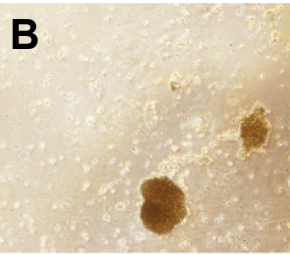

Postirradiation $160 \mathrm{~Gy}$

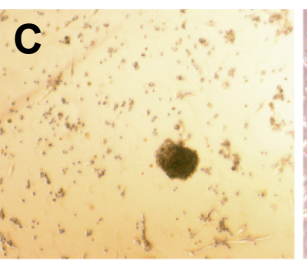

Post irradiation 240 Gy

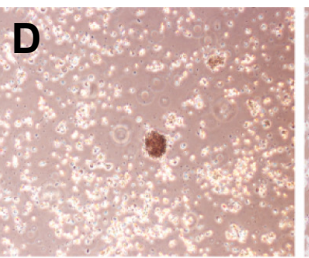

Post irradiation 320 Gy

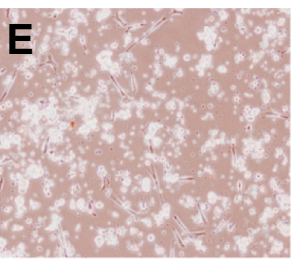

Figure S2 Diverse features of spheroid-bearing LL2 cells used as live attenuated vaccine, prepared after being subjected to 80 , 160,240 , and 320 Gy irradiation levels: alive yet unable to proliferate for safe vaccination.

Notes: (A) Before irradiation, the LL2 cells were expanded in DMEM/FI 2 and engaged in multicellular floating-spheroid mode with tumor-initiating cells or cancer stem cells transition. The spheroid-bearing LL2 was subjected to 80, 160, 240, and 320 Gy irradiation levels using RS-2000 biological irradiator (RadSource). (B and C) After irradiation at $240 \mathrm{~Gy}$ or lower levels, the spheroid-bearing LL2 cells revealed an acute irradiative shock by merging themselves into each other. (D) Irradiation at the 240 Gy level could keep the spheroid cells metabolically alive yet unable to proliferate, so the spheroid-bearing LL2 cells could be used as safe vaccines. (E) Yet, irradiation at the 320 Gy level could not keep the spheroid cells alive. $\times 100$ magnification.

Abbreviations: DMEM, Dulbecco's Modified Eagle's Medium; LL2, Lewis lung carcinoma.

OncoTargets and Therapy

\section{Publish your work in this journal}

OncoTargets and Therapy is an international, peer-reviewed, open access journal focusing on the pathological basis of all cancers, potential targets for therapy and treatment protocols employed to improve the management of cancer patients. The journal also focuses on the impact of management programs and new therapeutic agents and protocols on

\section{Dovepress}

patient perspectives such as quality of life, adherence and satisfaction. The manuscript management system is completely online and includes a very quick and fair peer-review system, which is all easy to use. Visit http://www.dovepress.com/testimonials.php to read real quotes from published authors. 